\title{
Consumo de drogas dentro del entorno "burgués": Influencia del control social formal e informal
}

\author{
Kemmesies, Uwe E* \\ *Doctor en Filosofía. Johan Wolfgang Goethe - Universität. Instituto de Pedagogía Social y de Formación de Adultos. \\ Enviar correspondencia a: \\ Uwe Kemmesies. Instituto de Pedagogía Social y de Formación de Adultos.Apartado de Correos 11 19 31. 60054 Francfort. \\ Tel.+069/798 23412 Fax: +069/798-28296 Correo electrónico: kemmesies@em.uni-frankfurt.de
}

\section{RESUMEN}

El presente estudio muestra los primeros resultados de una investigación sobre el ámbito del consumo socialmente integrado de drogas ilegales dentro de contextos socialmente establecidos, que es un campo poco investigado en Alemania. Se trata de estudiar la interacción entre el control formal (sistema penal y medicina) y el informal (mensajes procedentes del peer group, del círculo de amistades y conocidos)

Se entrevistan 34 consumidores de drogas ilegales, todos ellos socialmente integrados contactados a través de muestreo en 'bola de nieve'. Los resultados apuntan hacia dos hipótesis de trabajo:

- Dentro de la percepción de uno mismo, el consumo de drogas está subordinado a un "locus de control interno".

- Todo apunta a que existen mecanismos de control informales, cuyo efecto regulador se manifiesta por debajo del umbral de la ley general de prohibición de consumo de drogas.

Palabras clave: control social, consumo de drogas, influencia cultural, influencia pares, estudio cualitativo, Alemania.

\section{SUMMARY}

This study provides an overview of the socially-integrated use of illicit drugs in established social groups, something not very investigated until now in Germany. The main focus is on the process of interaction between formal (law and socialmedical control system) and informal (drug use messages of the peer group).

During the pilot study 34 socially integrated users of various illicit drugs, recruited via the snow ball sampling technique, were interviewed. The observations and findings made so far could be summarised in form of two working hypothesis:

- In the self perception drug use is obviously perceived as being mainly regulated by an internal locus of control.

- Even in the cases of consumption of illicit drugs in spite of the existing drug laws, the illicit status of certain substances seems to be of regulative impact on drug using behaviour; There seems to be informal control mechanisms associated with the legal status of the substances which work under the threshold of the drug laws' claim of General deterrence.

Key words: social control, use of drugs, qualitative study, cultural influence, peer influence, Germany.

\section{INTRODUCCIÓN}

A pesar de que, debido a la falta de investigación de este campo, no se pueden indicar relaciones exactas, se puede partir de la base, que una gran parte de los consumidores de drogas ilegales no desemboca (aún) en formas de consumo de drogas que a) puedan tener efectos negativos, es decir, problemáticos, sobre cuestiones vitales del día a día y b) derive en un modelo de consumo que sea socialmente evidente y punible desde un punto de vista legal (Bühringer et alt, 1997). De todo ello se deriva una situación precaria en cuanto a la observación de este fenómeno social: tanto el acercamiento jurídico como el estudio fenomenológico desde un punto de vista médico social se orienta sobre todo al consumo evidente y problemático. La punta del iceberg se ha convertido en el punto de orientación del control social y político de este fenómeno, cuando, en realidad, el conocimiento de la masa invisible de un consumo de drogas, que goza de la discreción social, integrada y, que, en gran medida, no crea excesivos problemas, ofrecería una práctica política que haría justicia al fenómeno siendo, además, un acercamiento que reflejaría la realidad diaria. Hasta la fecha, sola-

'Este artículo ha sido ya publicado en la revista Sucht 46,2 (2000) páginas 101-110 y su reproducción ha sido autorizada. 
mente tenemos una imagen muy vaga de los mecanismos de control del consumo discreto, "controlado" de drogas. El estado actual de la investigación apenas si permite una valoración precisa de la influencia del control social en general así como de la esfera jurídica en especial en lo que respecta a la aplicación de la ley sobre sustancias estupefacientes, la cual ha sido ampliamente debatida en los últimos años. Las líneas de investigación aquí señaladas se unirán en este trabajo a los objetivos proyectados de la investigación, centrándonos de modo sistemático - único en Alemania en cuanto a su alcance - en un "segmento positivo" (consumidores integrados socialmente) de la población consumidora.

El proyecto desea adquirir un conocimiento realista de las condiciones y los motivos del consumo de drogas dentro del "entorno burgués"; "entorno" se define en este trabajo de forma negativa, en la medida en que el campo de investigación se sitúa fuera del ámbito del consumo de drogas restringido a los drogodependientes, un sector de investigación que apenas si ha recibido atención. En este trabajo, este amplio sector se desplaza al centro de la atención investigadora. La idea que dirige el presente trabajo de investigación es la cuestión de la relación de importancia de instancias formales e informales de control social sobre el consumo de drogas.

En el presente artículo se presentan los primeros resultados de una primera fase piloto que finalizó en noviembre de 1998. No hace falta mencionar que, teniendo en cuenta el estado actual de los análisis realizados hasta la fecha - y la naturaleza de los proyectos piloto - las observaciones tienen todavía una calidad "ad-hoc" poco satisfactoria, que todavía requieren un fundamento empírico y analítico a establecer en el futuro desarrollo del proyecto.

\section{Metodología}

\subsection{Campo de investigación empírico y muestreo}

En nuestra intención de identificar mecanismos de control sociales que se centren de forma específica en las drogas y, en su caso, de definir formas de consumo asociadas con las drogas ilegales, se debía proceder en un primer momento a tomar contacto con 50 consumidores de cannabis, éxtasis, cocaína y opiáceos, respectivamente. Se planeó crear un muestreo paralelo de 50 personas que no hubieran consumido drogas ilegales hasta el momento, como grupo de comparación. El muestreo se realizó de conformidad con estos criterios:

\section{Criterios de exclusión \\ 1. Personas sin domicilio fijo}

2. Personas sin formación profesional completada

3. Personas que, durante el pasado año, no realizaron ningún tipo de actividad regulada - siendo la condición el haber existido durante al menos tres meses alguna relación laboral y / o alguna relación de ampliación / complemento a la formación

4. Personas que, debido a su consumo de drogas se hayan sometido a tratamiento terapéutico profesional

5. Personas con domicilio en la zona del Rin Meno.

\section{Criterios de selección}

6. Haber consumido durante un mínimo de diez ocasiones aquellas drogas que se han establecido como criterio de inclusión en un determinado grupo

7. Haber consumido durante el pasado año al menos una vez aquella sustancia que se ha establecido como criterio de inclusión en un determinado grupo

\section{Criterios de inclusión}

8. La inclusión en un determinado grupo se orienta en la sustancia que contenga la dinámica farmacológica más fuerte, que la persona consume (consumía) actualmente (a lo largo del pasado año)

El hecho de que, en comparación con otros proyectos de investigación tanto nacionales como internacionales, la trama de los criterios de muestreo sea tan estrecho debe - tal y como está previsto - garantizar el acceso al sector positivo objeto de la investigación, compuesto por consumidores de drogas ilegales integrados socialmente. Resulta obvio que este método de proceder conlleva asimismo un planteamiento "selectivo" del campo fenomenológico, un planteamiento que se desarrolla más allá de un consumo problemático, en conflicto con el entorno social. Por ello hay que subrayar nuevamente, que el valor informativo del proyecto de investigación "solamente" puede reflejar una parte del fenómeno "consumo de drogas ilegales", una parte que, sin embargo, hasta la fecha, no ha atraído la suficiente atención. Hasta la fecha, el reclutamiento de las personas entrevistadas se realizaba dentro del círculo de conocidos de los colaboradores del proyecto y mediante personas a las que se "había conocido casualmente" en cafeterías y bares, así como utilizando el método de muestreo de la bola de nieve (más detalladamente en: Kemmesies 2000). Cuando se trata - como en este caso - de conseguir acceso a contextos sociales muy sensibles en cuanto a la protección de datos personales y que, además, implica patrones de conducta ilegales, este método resulta prácticamente el único método viable. 
Muchos proyectos de investigación tanto nacionales como internacionales (remitimos al estudio Technical Working Group on Snowball Sampling and other Methods, 1997) han aplicado, en ocasiones, lamentablemente de forma poco crítica, este procedimiento especial de muestreo en estudios sobre el consumo de drogas de orientación etnográfica, estudios que se centran en contextos vitales que se encuentran fuera del estudio científico por parte de las instituciones y que son conscientes de la característica de "campo oscuro" de este campo de investigación.

Mediante este método, se corre el riesgo de caer en un así denominado "efecto de selección", ya que las cadenas informativas basadas en el método "de boca - a boca" se emplean entre personas a las que les une no sólo una cierta proximidad social y de espacio, sino asimismo una afinidad en cuanto al estilo de vida. La cuestión que se plantea es si pueden surgir ciertos "dobletes" en cuanto al tipo de persona, que reflejen el espectro fenomenológico solamente de forma limitada, especialmente en muestreos más pequeños. Para eliminar este potencial peligro, se ha planteado la realización de un muestreo de gran envergadura, teniendo especialmente en cuenta el amplio instrumental de sondeo y de la especial "sensibilidad" del campo de investigación. Por otro lado, se utilizará, de acuerdo con los postulados de Cohen (1990, 64 y siguientes) $)^{2}$, un procedimiento de bola de nieve junto con "secuencias aleatorias", que no garantiza una muestra aleatoria pero que parece ser el mejor sistema para seleccionar usuarios de una forma representativa al máximo (ibid 64). Para cerrar este apartado, debemos llamar la atención sobre el hecho de que el método de la bola de nieve se utiliza en este contexto como "Método de búsqueda de caso" y no como un muestreo estadístico dada la característica de "campo oscuro" del campo de investigación. Dentro de la fase piloto, cada cuarto entrevistado fue reclutado utilizando el sistema de cadena informativa; esta participación se incrementaría a medida que el proyecto avanzaba.

\section{Instrumentos de sondeo}

Con vistas a la creación de un método de investigación basado en una pluralidad de métodos, se utilizará un proceso de entrevistas abierto y centrado así como una entrevista estandarizada apoyada por un cuestionario. El motivo para seguir esta metodología es poder definir datos específicos del ámbito vital, posibilitando al mismo tiempo la incorporación de análisis comparativos sobre la base de una colección de datos, todo ello de un modo más sistemático.

La entrevista abierta, inspirada en el método de la "entrevista centrada" (Merton/Kendall, 1979), se centra en la evolución del consumo de drogas de los sujetos de la investigación; las construcciones biográficas 0 , en su caso, las descripciones retrospectivas de sucesos pasados se producen dentro del marco temático del consumo de drogas.

La entrevista estándar se apoya en un cuestionario muy completo, que se elabora con los encuestados en un diálogo discursivo de "pregunta - respuesta" dentro del marco de una entrevista cara a cara. Además de la compilación de datos biográficos estándar, las preguntas están dirigidas hacia la evolución del

\footnotetext{
${ }^{2}$ La puesta en práctica se realiza del siguiente modo: se pide a los encuestados que escriban en una lista los nombres de todas las personas que conozca a las que se pueda aplicar los criterios de selección (indicando las iniciales del nombre o motes). De esta lista se eligen encuestados potenciales así como "personas de repuesto" siguiendo el principio aleatorio. Después se pide a la persona encuestada que se ponga encontacto con estas personas. Si ello no fuera posible, se acudirá a las personas de repuesto. En caso de que la toma de contacto también resulte infructuosa, se renunciará a esta cadena de personas. De acuerdo con este principio, de cada 3 personas se eligirá a una persona encuestada potencial y una persona de repuesto.

${ }^{3}$ La encuesta se orienta en el siguiente hilo conductor:

- Orden cronológico de la evolución del consumo: deberá tenerse especial cuidado en reconstruir la evolución del consumo de drogas de acuerdo con su orden cronológico (desde el inicio hasta la situación actual), es decir, desde las fases de consumo más intenso hasta las fases de menor consumo o fases de abstinencia.

- Interacción vida (laboral) diaria y consumo: ¿Qué mecanismos de control posibilitan la unión de consumo de drogas y actividad vital diaria?

- Consumo de / interacción del estatus ilegal de las drogas con la actividad diaria: ¿Cómo se puede ocultar el consumo de drogas al entorno social "familia", "empresa", círculos de amistados / conocidos"? ¿cómo se puede ocultar el consumo de drogas a las autoridades de investigación? En este punto, hay que prestar especial atención a la situación del inicio en el consumo de las drogas, ¿Qué relación tenía el consumidor con la prohibición del consumo de drogas?, ¿Por qué no tuvo influencia sobre el comportamiento del individuo - en el sentido de no consumir drogas?

- Teoría sobre el consumo de droga: "filosofía subjetiva sobre las drogas: al final de la entrevista, el encuestado deberá pronunciarse como experto en su ámbito vital y en su historia personal, preguntándole sobre su propia filosofía subjetiva sobre las drogas.
} 
consumo de drogas y hacia la práctica actual diaria. ${ }^{4}$ El objeto global del cuestionario es facilitar los análisis comparativos entre los subgrupos sobre la base de una compilación de datos cuyo contenido ha sido elaborado de forma sistemática y unitaria. El uso del cuestionario está asimismo motivado en un intento de prescindir de datos tales como cuestiones "cuantitativas" o datos "duros", tales como la frecuencia de consumo, edad de inicio en el consumo de drogas, etc.

\section{El muestreo - observaciones introductorias}

De acuerdo con los criterios de investigación sobre el consumo de drogas, el muestreo realizado en la fase piloto está compuesto por una tercera parte de consumidores de drogas "blandas" (cannabis: 11) y por dos terceras partes de consumidores de drogas "duras" (éxtasis: 5; cocaína: 17; heroína: 1). La mayor parte de los consumidores de cannabis cuenta además con experiencia en el consumo de distintas drogas duras. Por su parte, los consumidores de drogas duras consumen cannabis en mayor cantidad que el grupo de drogas al que se le asignó en un primer momento. En la mayor parte de los casos se trata de patrones de consumo con un comportamiento polivalente. De acuerdo con estos patrones de consumo, las diferentes sustancias se consumen en diferentes entornos sociales. El objetivo del método que sirve de base a este proyecto, un método que se basa en dividir a los consumidores de drogas en diferentes grupos, es lograr un acercamiento empírico basado en el orden del campo fenomenológico; la evolución de proyecto mostrará si se dibujan perfiles específicos del grupo al que pertenecen por el tipo de sustancia, como por ejemplo en forma de asociaciones selectivas de diferentes drogas con ciertos entornos, patrones de consumo y / o evoluciones del consumo de drogas.

\subsection{Datos biográficos estándar}

La edad media de los encuestados asciende a $32,7^{6}$ años, una media de edad muy por encima de la media en investigaciones cuyo campo de referencia comprende a personas muy involucradas en el consumo de drogas [por ejemplo, Thiel et alter $1997(28,7$ años); Verthein et alter 1998 (28,7 años)]. Incluso las cifras actuales de la "Estadística anual (1997) de las asociaciones de asesoramiento y de tratamiento para adictos en la República Federal de Alemania" (Simon et alter, 1998) sobre la edad media en personas que reciben el primer tratamiento y tratamiento de recidivas, investigación cuyo centro de interés científico se centra en las sustancias psicoactivas de UmiD $^{7}$ (sic), se encuentran muy por debajo de la edad media de nuestro muestreo. Llama la atención la diferencia de edad de los consumidores actuales de drogas blandas (35,3 años) y de los consumidores de drogas duras (31,5 años), una diferencia que asciende a 3,8 años. Esta diferencia de edad apoya las hipótesis elaboradas en otras investigaciones, que postula que el consumo de drogas ilegales supone un fenómeno transitorio en la edad adolescente, un fenómeno que parece estar ligado a la superación de conflictos en la evolución, conflictos típicos de esta fase de desarrollo. (Hurrelmann y Bruñidle 1997). El hecho de que también los consumidores de drogas blandas, a excepción de dos casos, cuenten con una experiencia con drogas duras (en ocasiones intensa), nos permite pensar en una evolución en el consumo de drogas ligado a ciertas etapas o fases, parece confirmar la hipótesis de que los consumidores, a medida que van llegando a la edad adulta, asumen patrones de comportamiento típicos de su edad. Todo apunta a que el consumo de drogas más duras se va reduciendo a medida en que se va produciendo una integración en la estructura profesional y social. El consumo de cannabis, una droga considerada por un amplio espectro social

\footnotetext{
${ }^{4}$ El cuestionario contempla amplias módulos de preguntas sobre las siguientes áreas: anamnesis del historial del consumo de drogas (ver nota al pie de página 14) - financiación del ámbito vital - vínculos sociales - distribución de las drogas - contacto con instituciones penales y de medicina social - factores de influencia formales e informales sobre el consumo de drogas - preferencias acordes al estilo de vida - valores, orientación política - imagen de sí mismo.

${ }^{5}$ El objetivo de utilizar las denominaciones "drogas duras" y "drogas blandas" no es crear valoraciones sobre los riesgos de consumo de las diversas drogas, ya que el potencial de riesgo depende del consumidor.

${ }^{6} \mathrm{SD}:$ 6,9; Mín.: 22-Máx: 47.

${ }^{7} \mathrm{~A}$ modo de ejemplo, se indica la edad de los hombres en tratamiento, que se encuentra muy por encima de los valores correspondientes de las mujeres

$\begin{array}{lcc} & \text { Primer tratamiento } & \text { Segundo tratamiento } \\ \text { Cannabis } & 24.1 & 29.3 \\ \text { Éxtasis } & 22.5 & 26 \\ \text { Cocaína } & 26.1 & 19.7 \\ \text { Heroína } & 25.8 & 29.5\end{array}$
}


como una droga desligada de la cultura, sigue siendo una "constante" dentro del comportamiento de consumo, junto con las drogas legales.

El grupo de consumidores entrevistado está compuesto por 13 mujeres (38\%) y 21 hombres (62\%). La proporción de los sexos es más equilibrada en aquellos campos de estudio que tienen como objetivo los consumidores adictos, en los que existe una participación femenina de entre el $25 \%$ al 33\%. De acuerdo con las estadísticas sobre los tratamientos ambulatorios de ayuda contra la drogadicción, la proporción de mujeres en los grupos de sustancias sobre las que se centra el estudio, únicamente asciende a un $20 \%$ (Simon et alter, 1998, 24).

Todas las personas entrevistadas cuentan con una formación escolar y, a excepción de dos personas, que a lo largo de su periodo de formación se hicieron autónomos, finalizaron una formación profesional. Tres de cinco personas entrevistadas (59 \%) han realizado las pruebas de selectividad alemanas ${ }^{8}$ - un porcentaje muy por encima de la media de la República Federal de Alemania - y dos de cinco personas entrevistadas $(41 \%)$ cuentan con una formación académica superior.

Con casi un 70\%, la mayoría absoluta de los encuestados son solteros - es decir, ni tan siquiera una persona de entre cinco está casada. Una proporción casi idéntica puede encontrarse en un gran número de estudios sobre el problema "consumo de drogas ilegales", estudios que incluyen poblaciones muy divergentes dentro del espectro de consumidores de cannabis, pasando por consumidores de cocaína socialmente integrados hasta consumidores de heroína compulsivos ${ }^{9}$. A la luz de tales observaciones, la hipótesis basada en estadísticas sobre el tratamiento ambulatorio y que postula que el gran número de solteros en el grupo de consumidores de opiáceos indica una "integración social más débil así como una mayor individualización de este grupo de personas en comparación con personas adictas al alcohol o en comparación de la población total" (Simon et alter $1998,23)$ debe someterse a revisión. Es posible que la presencia de este gran número de personas solteras sea una expresión de estilos de vida no convencionales o menos convencionales (que el consumo de drogas ilegales simboliza), y no tanto un símbolo de aislamiento social debido al consumo de drogas.

Todas las personas entrevistadas ejercen una actividad regulada. El espectro profesional abarca desde la persona encargada de la economía doméstica, pasando por artesanos hasta académicos con altos cargos administrativos y departamentales. Encontramos a personas del ámbito policial y jurídico, profesionales autónomos, diseñadores gráficos, artesanos, personas procedentes del sector de ayuda contra las drogas, así como artistas del mundo de la música y de la escultura. La mayor parte de los encuestados ostentan una posición laboral estable; la actividad ejercida 0 , en su caso, la relación laboral se mantiene desde hace 7,4 años.

Por ello no es de extrañar el hecho de que los alimentos de la mayoría de los encuestados se costee mediante el salario profesional que perciben (>90). Solamente tres de los encuestados percibe un sustento económico de sus parejas sentimentales. Simplemente echando un vistazo a los ingresos que perciben las personas entrevistadas, nos permite afirmar que gozan de una posición laboral establecida. Los ingresos netos del último mes asciende a una media de $3190 \mathrm{DM}^{11}$, es decir, los ingresos están muy por encima del salario medio de la población que reside dentro del campo geográfico del presente estudio, que asciende a $2570 \mathrm{DM}^{12}$.

\subsection{Sinopsis sobre el historial del consumo de drogas y patrones del consumo de las mismas}

Los encuestados cuentan con una media de 16 años de experiencia en el campo del consumo de drogas ilegales. Los historiales del consumo de drogas se caracteriza por interrupciones más o menos largas en el consumo de drogas ilegales. Sobre la base de los criterios de muestreo, podemos afirmar que tales pausas en el consumo de las drogas no está influenciado por mecanismos terapéuticos. Hasta la fecha, ninguno de los encuestados se ha sometido a terapias por su consumo de drogas - ni siquiera se han producido contactos de asesoramiento con organismos ambulatorios de ayuda contra la drogadicción. Ninguno de los encuesta-

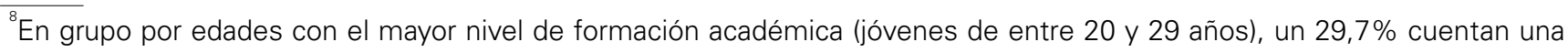
titulación de una escuela técnica superior o titulación académica (instituto nacional de estadística alemán 1997,70).

${ }^{9}$ Kleiber et alter $(1997,214)$, consumidores de cannabis - 89\%; Cohen (1990, 74); consumidores de cocaína- 84\%; Kemmesies $(1995,134)$, consumidores de heroína.

${ }^{10} \mathrm{SD}: 7,7$; Mín: 0,5 - Máx. 25

${ }^{11}$ SD: 1687; Mín: 900 - Máx. 8000

${ }_{12}$ Cálculos propios considerando los datos correspondientes a los ingresos medios anuales del año 1994 de las siguientes ciudades y comarcas: del área Renania - Meno: Francfort, Wiesbaden, Offenbach, Darmstadt o circunscripción del Meno - Taunus (Hessisches statistisches Landesamt, Oficina Regional de Estadística 1997, 18).
} 
dos considera necesario acudir en el futuro a un organismo de ayuda contra la drogadicción. Además de todo lo expuesto y considerando el estado actual de las valoraciones, no pueden formularse postulados "objetivables" acerca del consumo de drogas o estado de dependencia de tales sustancias, considerando el instrumental de diagnóstico utilizado.

La primera droga ilegal que los encuestados habían consumido es cannabis. En el caso de todos los encuestados, el consumo de esa droga ilegal venía precedida por experiencias en el consumo de drogas legales. Con dos excepciones, todas las personas entrevistadas pueden catalogarse como consumidores de cannabis experimentados; la mayoría absoluta (>70\%) contabiliza una frecuencia de más de 200 ocasiones en las que ha consumido la antes reseñada droga. Casi siete de cada diez encuestados han consumido en más de diez ocasiones cocaína en el pasado y cuatro de cada diez encuestados cuentan con una amplia experiencia en el consumo de 'éxtasis'. Además, una de cada cuatro personas entrevistadas tiene experiencia con la heroína, que no se reduce a un único consumo experimental; casi una de cada seis personas ha consumido, hasta la fecha, esta droga en más de diez ocasiones. Con respecto al consumo permanente a lo largo de toda la vida, únicamente dos de las personas - a excepción de las drogas legales - entrevistadas eran consumidores puros del cannabis.

Considerados en su totalidad, los encuestados pueden clasificarse en consumidores regulares hasta consumidores intensivos de drogas legales. Solamente cinco personas (15\%) indican ser no fumadores; un $60 \%$ de los encuestados fuma un mínimo de diez cigarrillos al día. Con un índice de un $85 \%$ de fumadores, el número asciende a más del doble que en el encuesta representativa realizada en todo el territorio federal sobre el consumo de sustancias psicotrópicas por parte de personas con edades comprendidas entre los 18 y los 59 años de edad (Kraus y Bauernfeind, 1998, 53). Solamente uno de cada diez encuestados indica beber menos de una vez al mes. Un 15\% de los encuestados indica consumir (casi) diariamente bebidas alcohólicas y otro $50 \%$ de los encuestados indican que consume alcohol más de una vez a la semana.
La mayor parte (85\%) de nuestros encuestados puede catalogarse como consumidores habituales de cannabis (un mínimo de un consumo mensual). Algo más de uno de cada cuatro encuestados indica que consume cannabis diariamente $(27 \%)$.

Después del cannabis, la cocaína es la droga ilegal más consumida, aunque la intensidad de su consumo es significantemente inferior. Casi uno de cada dos encuestados (48\%), que señalaron ser consumidores de drogas duras, había consumido cocaína en los últimos tres meses. De acuerdo con los criterios de clasificación de la encuesta representativa realizado por el Instituto de Investigación Terapéutica sobre el abuso de sustancias estupefacientes (ver: Herbst et alter $1995,9)$, alrededor de nueve de cada diez consumidores encuestados son consumidores habituales de cocaína (87\%), cuatro de cada diez (39\%) pueden catalogarse como consumidores de Éxtasis y de "Speed", y uno de cada cuatro personas encuestadas (26\%) son consumidores de LSD y más de dos personas de cada diez (43\%) encuestadas son consumidores de "hongos". Resulta significativo el consumo comparativamente intenso del grupo de sustancias biógenas (hongos psicoactivos).

Tal y como puede observarse en la intensidad del consumo, las drogas duras parecen ocupar un lugar marginal dentro de las pautas del consumo colectivo dentro de nuestro sondeo: ningún encuestado indica que consume este tipo de sustancias con una frecuencia diaria o con mucha frecuencia a lo largo de la semana.

\subsection{Sobre la influencia de instancias formales e infor- males de control social sobre el consumo de dro- gas.}

Consideremos en primer lugar los motivos así como el trasfondo psicosocial del estudio del consumo de drogas (Fig. 1)

Resulta llamativo la gran importancia que tiene el motivo de la "curiosidad" así como - a diferencia de la asociación del consumo de drogas con problemas personales, tesis que podemos encontrar en amplios sectores de la investigación sobre el consumo de drogas (sinopsis en: Hawkins et alter, 1992) - la importancia marginal de un trasfondo psicosocial problemá-

\footnotetext{
${ }^{13}$ El cuestionario abarca un instrumental para establecer el diagnóstico destinado al registro de la "adicción a la sustancia" con vistas a determinar los 12 meses anteriores a la celebración de la encuesta. El módulo de preguntas se basa en los criterios del DSM-IV y se orienta en la versión de Kleiber et alter (1997) Se han considerado los siguientes siete criterios del DSM-IV con vistas a determinar el grado de adicción: 1. Desarrollo de tolerancia, 2. Síntomas de deshabituación, 3. Un mayor consumo del que se había propuesto el consumidor; 4. Intentos de control del consumo; 5. Influencia de las actividades; 6. Consumo continuado a pesar del las posible secuelas.

${ }^{14}$ El predominio del motivo de la "curiosidad" no sorprende especialmente - se trata de un factor decisivo, tal y como lo demuestran estudios nacionales e internacionales (en sinopsis: Reuband, 1990, 103 ff'
} 


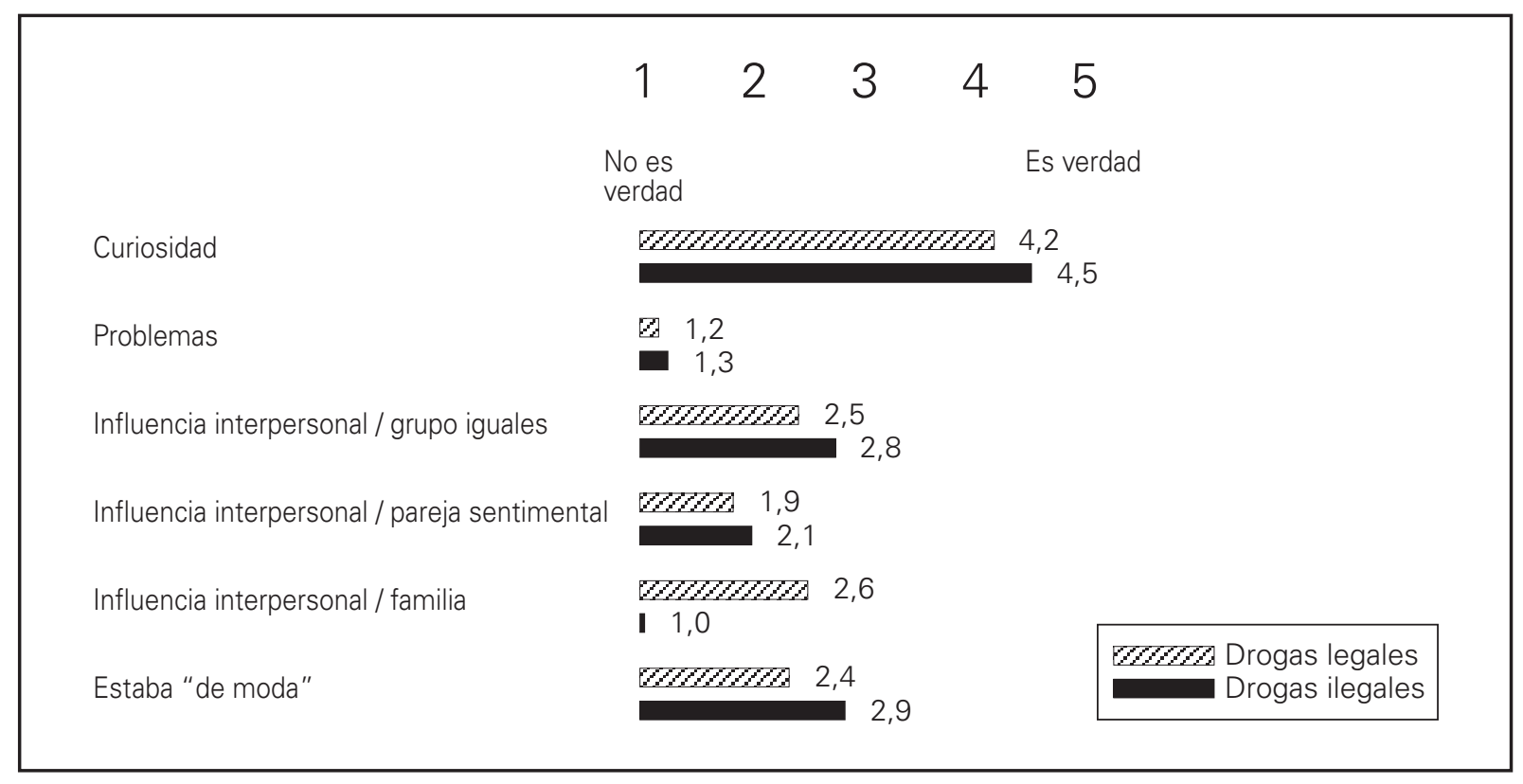

FIGURA 1. Motivos para iniciarse en el consumo de drogas legales e ilegales (n:34)

tico a la hora de iniciarse en el consumo de drogas legales e ilegales. En cuanto a las restantes categorías de motivos, podemos destacar diferencias relacionadas con las diferentes drogas. En tanto que los "motivos sociales" relativos a la influencia interpersonal a través de personas de la misma edad ${ }^{15}$ o del entonces compañero sentimental del encuestado tienen una importancia media, parece que la interacción dentro del entorno familiar tiene relevancia con la iniciación en el consumo de drogas. La categoría de unos motivos de orientación (sub)cultural, que podemos definir como "estaba de moda" parece tener una mayor importancia en lo que respecta a las drogas ilegales. Es posible que se perfile un "estatus" especial de las drogas ilegales, el cual convierte las mismas en un símbolo social así como del estilo de vida, en un símbolo de pertenencia a la nueva corriente social (sub)cultural. Cabe señalar, con ciertas reservas, que, a excepción del motivo social "familia", todas las categorías de motivos referentes a las drogas ilegales indican un mayor grado de consenso. Esto último puede interpretarse también como indicador del "estatus" cultural que ostentan las drogas ilegales: para traspasar el umbral de las drogas ilegales, se requiere un mayor esfuerzo y, por ello, un mayor grado de motivación con vistas a anular los mecanismos de control que rodean estas sustancias. Estos últimos se caracterizan por orientarse en normas y valores sociales.
En cuanto a los textos de control formales de la Ley de sustancias estupefacientes hay que destacar que, apoyando las tesis promulgadas por Wilkens et alter $(1997,425)$, más de nueve de cada diez entrevistados (94\%) eran conscientes del "estatus" jurídico de la sustancia antes de iniciar su consumo. A la luz de los resultados de nuestra encuesta, resulta obvio constatar que el "estatus" jurídico de las sustancias, recogido en la Ley de sustancias estupefacientes en cuanto a una normativa preventiva que rige el comportamiento de los individuos, no ha influido en las decisiones de los individuos, ya que se trata de consumidores de drogas ilegales. Más bien todo lo contrario: algunos encuestados señalan que dicha ley tuvo el efecto contrario, ya que la prohibición del consumo de sustancias consideradas ilegales tuvo un efecto potenciador del factor "curiosidad", considerándose, por tanto, un factor catalizador de la categoría que describe los motivos del consumo de sustancias. Dicho factor "curiosidad" tiene un efecto importante sobre el comportamiento. Resulta sorprendente, sin embargo, que, en comparación, la prohibición del consumo de drogas tiene menos importancia a la hora de iniciarse en el consumo de drogas: de las 16 encuestas transcritas y analizadas desde el punto de vista de las historias vitales experimentadas hasta el momento, nos encontramos con que la prohibición del consumo de drogas y las respectivas instancias de control

\footnotetext{
${ }^{15}$ Esta constelación de motivos apunta, tras una primera categorización de las encuestas orientadas en la biografía, a la función psicosocial del consumo de drogas, expresada en un acceso más fácil a círculos de personas de la misma edad o, en el caso de relaciones de pareja, en simbolizar una cercanía social así como una cercanía en cuanto a experiencias vitales.
} 
del aparato policial se menciona, como mucho, de pasada. El proceso de iniciación parece no requerir técnicas especiales de "neutralización"; tampoco requiere esfuerzos especiales de neutralizar las estructuras normativas acerca del consumo de drogas: de acuerdo con la teoría de Sykes / Matza (1968), los consumidores actualizan, sin especial esfuerzo, argumentos y aspectos que restan importancia a tales circunstancias. Estos argumentos y aspectos que crean, "una vez racionalizados a priori o a posteriori, momentos de inocencia subjetiva" (Moser, 1987). Conscientes del "estatus" jurídico de las drogas, la iniciación en el consumo de drogas se considera una violación de las normas que no viene acompañada por un sentimiento de culpa. Tampoco se puede constatar que la prohibición del consumo de drogas forme disociaciones cognitivas que el individuo experimente como condicionantes restrictivas de su comportamiento, aunque haya que matizar este punto en el sentido de que las posibles disonancias cognitivas inducidas por la prohibición, son repelidas por el comportamiento colectivo así como por la escala de valores que rige a un grupo de personas de la misma edad.

Considerando que el objeto del estudio es el entendimiento de pautas de comportamiento, las respuestas a la pregunta abierta acerca de las dos instancias más influyentes relativas a la falta de consumo de determinadas drogas ilegales resultan sorprendentes: ni un solo encuestado menciona expresamente las instancias de control del ámbito penal y de la medici- na social - ni la prohibición del consumo de drogas ni las informaciones procedentes de entidades de ayuda a la drogadicción se consideran influencias de carácter relevante. Por el contrario, en la mayoría de los casos, los encuestados indican que ninguna de estas instancias ha sido un factor decisivo a la hora de probar o de consumir un determinado tipo de droga; es más, y así lo confirman los comentarios de los encuestados a esta pregunta, parece que la decisión sobre el consumo de diversas sustancias está supeditada al autocontrol, y, en menor grado, a los factores de control existentes en su entorno social. Las respuestas ofrecidas a la categoría "información a través de los medios" parece confirmar esta imagen, nombrada por una de cada cuatro personas encuestadas. El motivo es que la información ofrecida a través de los medios solamente se puede percibir de manera receptiva y no están asociados a un determinado control del comportamiento, ya que no se ofrecen dentro de un contexto de interacción de carácter personal. Mantienen abiertas las diversas opciones de comportamiento, pudiendo ser utilizadas como impulsos potenciadores de los propios patrones de opinión, de valores y de comportamiento.

Además del autocontrol aparece el mesosistema o, en su caso, los microsistemas integrados por el círculo de amigos, de pareja y familiar que lo integran como factores determinantes sobre el consumo de las drogas.

La "experiencia personal" se considera, con diferencia, como la fuente de información personal más

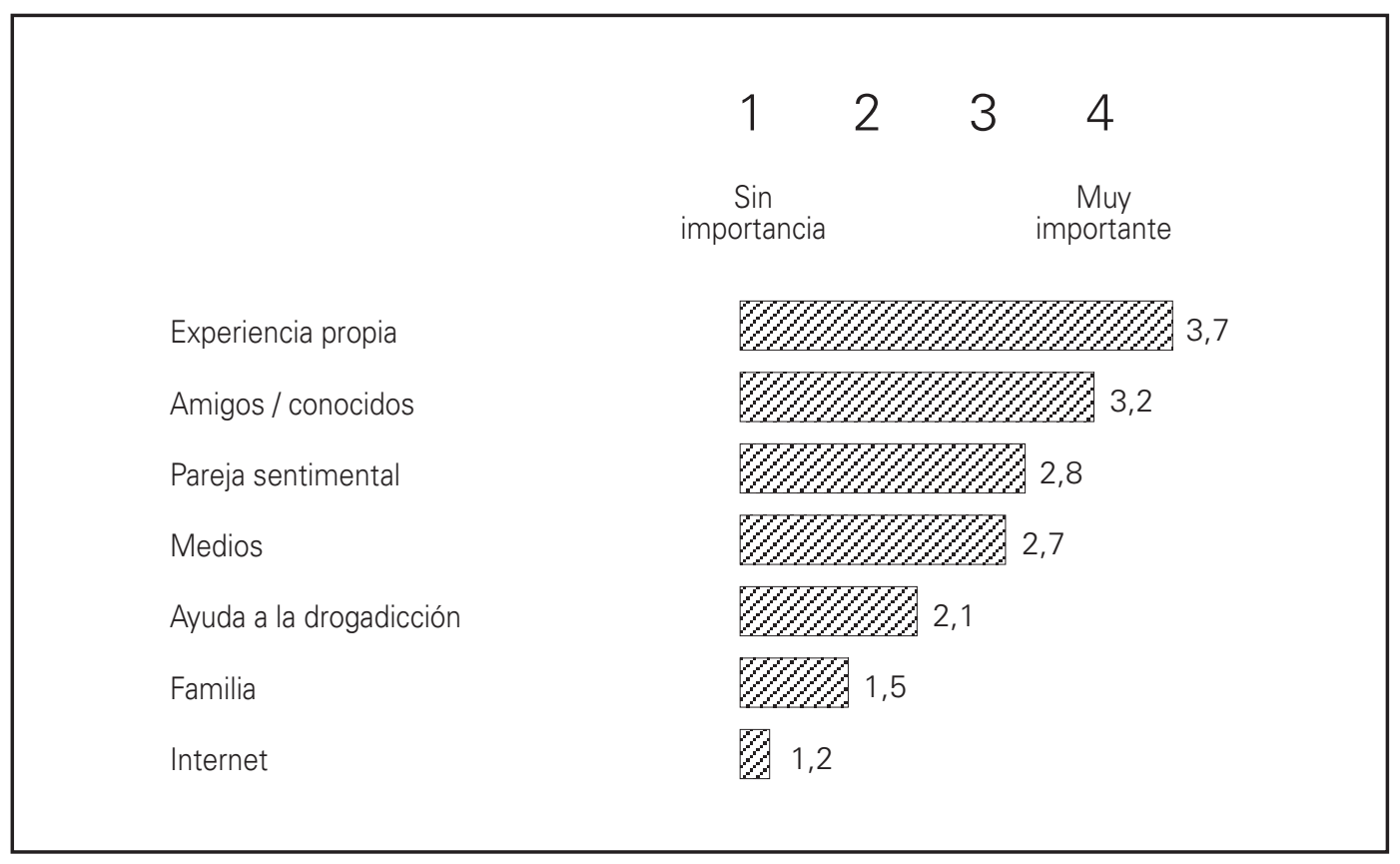

FIGURA 2. Influencia de diversas fuentes de información sobre drogas ilegales (n:34) 
importante en cuanto se refiere al consumo de drogas $^{16}$. De acuerdo con las opiniones expresadas fuera del contexto de la encuesta, la experiencia personal no se limita a experiencias personales relacionadas con el consumo de drogas, sino también es extensible a observaciones realizadas dentro del contexto social más próximo, en forma de experiencias ajenas con relevancia sobre el comportamiento. En consonancia con los resultados obtenidos en diversos trabajos de investigación, que reflejan la enorme importancia que tienen los grupos de referencia compuestos por personas de la misma edad ${ }^{17}$ en la evolución del consumo de drogas, nuestros encuestados dan mucha importancia al entorno social más cercano integrado por amistades, conocidos y de pareja. Asimismo, los medios de comunicación también se consideran factores importantes de influencia y, en su caso, de fuente de información, mientras que la ayuda contra la drogadicción, la familia e Internet ejercen una influencia prácticamente nula.

\section{Un primer resumen}

Las observaciones realizadas dentro de esta fase piloto pueden reducirse a tres hipótesis de trabajo relacionadas con el objeto de estudio del presente trabajo, hipótesis que deberán analizarse con más profundidad en los subsiguientes estudios sobre la base de un mayor número de casos y de análisis comparativos más diferenciados.

- Todo parece indicar, que el consumo de drogas, entendido como un comportamiento subjetivo íntimamente ligado al individuo, está dirigido de forma importante por mecanismos de autocontrol, es decir, en la consciencia del individuo, entendida como una autoatribución, queda supeditada a un "locus de control interno".

- El entorno social compuesto por un control informal a nivel del mesosistema (entendido como confi- guración individual de diversos microsistemas - especialmente: el círculo de amigos, conocidos, familiar) parece ejercer una influencia de enormes proporciones sobre el comportamiento del individuo en cuanto al consumo de drogas, en comparación con el control formal a nivel del exosistema (sistemas de ayuda contra la drogadicción, escuela, instancias de acción penal) y del macrosistema (ley sobre sustancias estupefacientes).

- No obstante, algunas observaciones apuntan a que - por debajo del nivel de la medida preventiva contenida en la ley sobre sustancias estupefacientes dirigida a evitar el contacto del individuo con las drogas ilegales -a la categoría legal de dichas sustancias se une una influencia de control informal. Debido al poder desacreditador de los consumidores de drogas, la preferencia por las drogas ilegales se oculta ante una parte del entorno social, para evitar una posible estigmatización ${ }^{19} \mathrm{O}$, incluso, las sanciones negativas. Todo parece indicar, que no se debe poner en juego la existencia "burguesa". En este sentido, las reacciones observables así como las reacciones anticipadas del entorno social, ejercen influencia sobre la forma del consumo de drogas ilegales. El consumo de drogas se practica dentro de un entorno social selectivo y está dirigido por las diversas situaciones sociales, teniendo en cuenta la composición social del "setting social" y atendiendo al grado de adecuación de la situación (¿con quién y cuándo se consume?). Dentro de aquellos entornos sociales, en los que la persona percibe que el consumo de drogas ilegales no cuenta con la aprobación de los integrantes de ese mismo entorno, sino que se decanta por la regulación legal y social del macrosistema que desaprueba y rechaza esta práctica, el individuo no practica su consumo y tampoco se tematiza. Los datos parecen apuntar a que el comportamiento del individuo con relación al consumo de drogas solamente se muestra hacia el exterior si no caben esperarse consecuencias negativas para su posición social.

\footnotetext{
${ }^{16}$ Una observación, que también se constató en un estudio realizado en Gran Bretaña: "Respondents said they themselves were the biggest influence which had dissuaded them from using drugs and routes they never had used, and had led to discontinued use of those they had"

${ }^{17}$ Comparar, por ejemplo, los siguientes estudios: Reuband 1992; BzgA, 1994; Lachnitt/Kampe, 1996 ; Fountain et alter, 1997.

${ }^{18}$ La diferenciación entre macro, eso, meso, y microsistemas se basa en la construcción teórica de la "estructura del setting en el consumo de drogas", surgida de modelos teóricos de Zinberg y Bronfenbrenner (más ampliamente: Kemmesies 1995).

${ }^{19}$ Ello se desprendía claramente de las respuestas a las preguntas sobre el "setting" favorito con relación al consumo de drogas: mientras que la mayoría absoluta de los encuestados consume drogas en los círculos anónimos que se crean en viviendas particulares, (69\%), preferían consumir drogas legales dentro de locales de consumo (semi)públicos. Esta teoría se ve apoyada por la declaración de los encuestados, que indican consumir drogas ilegales (56\%) dentro de círculos de personas de confianza, como regla de control del consumo. La preferencia de habitáculos privados destinados al consumo de drogas ilegales garantiza un control sobre el comportamiento que se desvía de la norma, ya que se puede fijar el grado de publicidad. Ya que el "setting" físico de los habitáculos privados garantiza al tiempo un control sobre la composición del "setting" social.
} 


\section{AGRADECIMIENTOS}

La fase piloto del proyecto "Sobre el precario ámbito de vigencia de la ley sobre sustancias estupefacientes: el consumo de drogas ilegales dentro del entorno burgués" ha sido financiado por la fundación Volkswagen. En agosto de 1999 se aprobó la continuación de la investigación por un periodo de tres años. En septiembre de 2002 se presentará el informe final.

\section{BIBLIOGRAFÍA.}

Bühringer, G., Adelsberger, F., Heinemann, A., Kirschner, J., Knauß, I., Kraus, L., Püschel, K. und Simon, R. (1997). Schätzverfahren und Schätzungen 1997 zum Umfang der Drogenproblematik in Deutschland. Sonderheft 2. Sucht, 43.

BZgA (1994). Die Drogenaffinität Jugendlicher in der Bundesrepublik Deutschland. Wiederholungsbefragung 1993/1994. BZgA, Köln.

Cohen, P. (1990). Drugs as a social construct. Elinkwijk, Utrecht.

Fountain, J., Bartlett, H., Griffiths, P., Gossop und M., Strang, J. (1997). The process of re-sistance to drug use among young people exposed to drugs. Final report. National Addiction Centre, London.

Hawkins, J.D., Catalano, R.F. und Miller, J.Y. (1992). Risk and protective factors for alcohol and other drug problems in adolescence and early adulthood: implications for substance abuse prevention. Psychological Bulletin, 112: 64-105.

Herbst, K., Kraus, L., Scherer, K. und Schumann, J. (1995). Repräsentativerhebung zum Gebrauch psychoaktiver Substanzen bei Erwachsenen in Deutschland. Telefonische Erhebung 1994. IFT, München.

Hessisches Statistisches Landesamt (1997). Das verfügbare Einkommen der privaten Haushalte in Hessen 1989 bis 1994. Hessisches Statistisches Landesamt, Wiesbaden.

Hurrelmann, K. und Bründel, H. (1997). Drogengebrauch Drogenmißbrauch. Primus, Darm-stadt.

Kemmesies, U.E. (1995). Kompulsive Drogengebraucher in den Niederlanden und Deutschland. Die offenen Drogenszenen in Amsterdam und Frankfurt a.M. VWB, Berlin.

Kemmesies, U.E. (1999). Snowball Sampling. In: EMCDDA (ed.): Understanding and Responding to Drug Use: The
Role of Qualitative Research. EMCDDA Monograph Series. EMCDDA, Lissabon: 265-271.

Kleiber, D., Soellner, R. und Tossmann, P. (1997). Cannabis-konsum in der Bundesrepublik Deutschland: Entwicklungs-tendenzen, Konsummuster und Einflußfaktoren. SPI, Berlin.

Kraus, L. und Bauernfeind, R. (1998). Repräsentativerhebung zum Gebrauch psychoaktiver Substanzen bei Erwachsenen in Deutschland 1997. Sucht, Sonderheft 1.

Lachnit, G. und Kampe, H. (1996). Konsumorientierung und Suchtgefährdung. Sucht, 1: 6-19.

Merton, R.K., Kendall, P.L. (1979). Das fokussierte Interview. In: Hopf, C., Weingarten, E. (Hg.): Qualitative Sozialforschung. 1. Aufl. Klett-Cotta, Stuttgart: 171-204.

Moser, T. (1987). Jugendkriminalität und Gesellschaftsstruktur. Suhrkamp, Frankfurt am Main.

Reuband, K.-H. (1990). Soziale Determinanten des Drogengebrauchs. Habilitationsschrift. Fakultät der Wirtschaftsund Sozialwissenschaften/Universität zu Köln, Köln.

Reuband, K.-H. (1992). Der Mythos vom einsamen Drogenkonsumenten. Kontakte zu Gleichaltrigen als Determinanten des Drogengebrauchs. Sucht, 3: 160-172.

Simon, R., Palazetti, M., Bühringer, G., Schmidtobreick, B., Heleas, I. und Hüllinghorst, R. (1998). Jahresstatistik 1997 der ambulanten Beratungs- und BehandlungssteIlen für Sucht-kranke in der Bundesrepublik Deutschland. EBIS-Berichte, Bd. 29, Frei-burg/Hamm /Kassel/ München.

Statistisches Bundesamt (1997). Datenreport 1997. Bd. 340. Bundeszentrale für politische Bildung, Bonn.

Sykes, G.M. und Matza, D. (1968). Techniken der Neutralisierung: Eine Theorie der Delinquenz. In: Sack, F. und König, R. (Hg.): Kriminalsoziologie. Akademische Verlagsgesell-schaft, Frankfurt am Main: 360-371.

Technical Working Group on Snowball Sampling and other Methods (1997). Handbook on Snowball Sampling. Pompidou Group - Council of Europe, Straßburg.

Thiel, G., Friedrich, E. und Wiese, K. (1997). Aspek-te der Lebenswirklichkeit, Nutzung von Drogenhilfeeinrichtungen sowie drogenpolitische Forderungen von 323 Personen der offenen Hamburger Drogenszene. WZfS, 1/2: 35-41.

Verthein, U., Degkwitz, P., Kühne, A. und Krausz, M. (1998). Komorbidität von Opiatabhängigkeit und psychischen Störungen. Sucht, 4: 232-246.

Wilkens, W., Thiel, G., und Friedrich, E. (1997). Ecstasy: Bedeutung des rechtlichen Status, Konsumwirkungen und Konsumhäufigkeit. Sucht, 6: 422-429. 